(C) [2010] IEEE. Reprinted, with permission, from Didar Zowghi, A Framework for the Elicitation and Analysis of Information Technology Service Requirements and Their Alignment with Enterprise Business Goals, Computer Software and Applications Conference Workshops (COMPSACW), 2010 IEEE 34th Annual, 19-23 July 2010. This material is posted here with permission of the IEEE. Such permission of the IEEE does not in any way imply IEEE endorsement of any of the University of Technology, Sydney's products or services. Internal or personal use of this material is permitted. However, permission to reprint/republish this material for advertising or promotional purposes or for creating new collective works for resale or redistribution must be obtained from the IEEE by writing to pubspermissions@ieee.org. By choosing to view this document, you agree to all provisions of the copyright laws protecting it 


\section{A Framework for the Elicitation and Analysis of Information Technology Service Requirements and Their Alignment with Enterprise Business Goals}

\author{
Didar Zowghi \\ School of Software \\ Faculty of Engineering and IT \\ University of Technology, Sydney (UTS) \\ Australia \\ Didar.Zowghi@uts.edu.au
}

\author{
Zhi Jin \\ Key Laboratory of High Confidence Software \\ Technologies \\ Peking University \\ Beijing, P.R. China \\ zhijin@sei.pku.edu.cn
}

\begin{abstract}
As the economies of the world have become increasingly dependant on Information Technology (IT) services, there is a need for service designers and developers to focus on co-value creation between service providers and service consumers. Developers need to conduct a more rigorous and systematic identification, elicitation, and analysis of IT service requirements than ever before so that the resulting IT services are closely aligned with the enterprise business requirements. Research in Services Science from the business and management discipline has mostly focused on the delivery and management of services experience from the business perspective. Much of the research focus in Service Oriented Computing (SOC) so far has been on the design and delivery of services (especially Web Services), but engineering of IT service requirements has received much less attention. The overall aims of the proposed research is the design and development of an integrated framework and its supporting toolset for the systematic identification, elicitation, and analysis of IT service requirements that satisfy consumers' needs and are closely aligned with their enterprise business goals.
\end{abstract}

Keywords- service requirements; elicitation; analysise; alignment;

\section{INTRODUCTION}

Service industries have dominated the established economies of the world for many years. Services are ubiquitous and are a critical element of the modern world economies. They play an increasingly important role in most industry sectors including Information Technology. Although the study of services has a long history in the Business, Management and Marketing disciplines, more recently services have also emerged as a new field of study in computer science. In 2004, IBM initiated a new academic discipline named Services Science, Management, and Engineering (SSME)[1]. SSME is a multi-disciplinary research field that integrates aspects of computer science, operations research, engineering, management, business, social and cognitive sciences. The primary goal of SSME is creation of value to the organizations through their IT operations function. A secondary goal is the alignment of services with the business strategy for service consumers.
Whilst IT services providers' aim are for achieving cost efficiency and optimization in operational processes, IT service consumers increasingly demand flexibility and agility to evolve ongoing IT services according to changing enterprise business needs. Two of the main frameworks in IT service industry addressing these demands are the Information Technology Infrastructure Library (ITIL) [3] and the Control Objectives for IT (COBIT) [6] ITIL is often referred to as a collection of best practices that also exists as an international standard variant, ISO 20000 [5]. ITIL helps organizations to understand the business value that IT services provide to internal and external stakeholders. Understanding this value is crucial for the definition of Service Level Agreements (SLA) between an IT department and its stakeholders. However, ITIL does not define how this value is to be elicited and co-created with the stakeholders. Neither does it provide a systematic and rigorous approach for analyzing the enterprise business domain. COBIT is an IT governance framework and unlike ITIL that addresses Service Management directly, COBIT defines the organizational structure and metrics for IT departments. This paucity of methods, processes and tools within the current best practices of IT service management thus creates an opportunity for the exploration of research results from the Requirements Engineering discipline for application within Services Science [4].

ITIL articulates several important challenges faced in service management and states several generic guidelines and claims that experience has shown these guidelines may help overcome them[3]. Examples of these challenges are "unclear or changing requirements from the business", "lack of awareness and knowledge of service and business targets and requirements", "poor relationship, communication or lack of cooperation between IT service provider and the business which may result in the design not achieving the business requirements", and "understanding the business requirements and the business priorities". It is not surprising that these challenges are almost identical to those that have been faced by software developers over the last 4 decades and many of them have been extensively and successfully addressed by the RE researchers and practitioners. Unlike 
RE results, ITIL does not provide a systematic method, well-defined processes or supporting tools for the elicitation, analysis and design of IT services' requirements. This motivates us to examine the application of RE research results to address these challenges identified in the IT services, that is the focus of our research proposal.

The proposed research project will:

(a) Develop an approach based on domain modeling for eliciting requirements of the IT services, improving the quality of these services as well as the collaboration patterns for the service delivery, from both the high-level business strategies and the consumers' intentions (Task I).

(b) Develop a novel goal-oriented service centric requirements analysis approach (Task II) that can also take multi-viewpoints of service consumers into account. This is fundamentally different from existing work on goal orientation, which primarily focuses on single goal-tree, but ignores the intentions from multiple consumers.

(c) Develop an innovative IT services change process for aligning business strategy and services (Task III). The results will significantly advance the RE process for IT services both in research and practice.

(d) Develop a prototype toolset to support the proposed integrated framework. The tool will also be used in evaluating and validating the proposed approach (Task IV).

The tool-based evaluation of the framework will provide a real-world case study that can be used as a benchmark for effective IT services discovery and design in practice.

\section{REQUIREMENTS ELICITATION}

Collaborative approaches to requirements elicitation are well known especially facilitated workshops, where the different stakeholder groups are represented and work together to collectively conduct the process of requirements elicitation. These techniques have been found to be very successful in not only producing quality requirements, but also in achieving buy-in by stakeholders and instilling project ownership [7]. One of the obvious advantages of using group workshops is the ability to integrate other elicitation techniques into them, and to incorporate their combined usage into a defined requirements process [8].

In earlier work we have developed a collaborative and situational method (OUTSET) [9] and its supporting tool (MUSTER) [10], specifically for requirements elicitation workshops, which utilizes, extends, and demonstrates a successful application of intelligent technologies for Computer Aided Software Engineering and Computer Aided Method Engineering. In this project we intend to initially apply OUTSET and MUSTER to IT service requirements elicitation and if needed, extend it to cater for specific characteristics of IT services. Recent results obtained in Collaboration-based requirements elicitation from the multiple domain experts [28] are complementary to OUTSET and will be further extended in this project.

\section{ANALYSIS OF IT SERVICE REQUIREMENTS}

Human interactions, experiences and sense making that occur in the development and delivery of IT services provide significant challenges, especially when it involves multiple actors, with differing world views, goals, logic, assumptions, and experiences. Thus conflicts are likely to occur during service requirements discovery and analysis. We draw upon our extensive previous research on "managing inconsistencies in RE" ([15][16][17]) to develop analysis methods and supporting tools for analyzing conflicts and inconsistencies among IT service requirements. $I^{*}$ framework [14] and Problem Frames (PF) method [2] are among the most significant RE analysis approaches to date. In $i^{*}$ framework requirements are understood in terms of goals, and the overall objective is to seek answers to the "why" question in the design of a system. I* starts with high level, composite goals of the stakeholders, and then refines them into hierarchical goal structures. It also models social dependency relationships that future system actors have to handle. The PF approach suggests new and extensible mechanisms for problem analysis. The problem analysis considers that software is used to address the problems in the real world. It represents requirements as expected phenomena within problem domains, such that their explicit treatment can be given to the constraints originated from the problem context. In our previous work ([11][12][13]), we have defined the capability model of Web services based on the environment ontology. We draw upon these results from business service design to supply ontological domain models and service problem frames. These domain models and problem frames will become the guidelines for capturing the customers' needs for business services that are aligned with enterprise business goals and strategies.

\section{APPROACH AND METHODOLGY}

The conceptual view of the four main research tasks is shown in Figure 1.

\section{Task I: Elicitation of requirements for IT services}

Different from software requirements, IT service requirements are implied in the enterprise value strategies, and are situated in the interactions with the enterprise partners and the basic IT infrastructure, i.e. the enterprise interactive environment, for supporting value co-creation. Thus, in requirements elicitation for IT services we need to pay attention to the enterprise strategies and the interactive environment. Moreover, value co-creation implies that the requirements will come from all the participants not only the service providers. Requirements elicitation for IT services needs significant shared understanding and has to be conducted in a more collaborative manner than software requirements. Thus, we plan to focus on the following two sub-tasks for developing a systematic and collaborative IT service requirements elicitation approach. 


\section{Task I-A. Developing Ontological Domain Model and} Service Problem Frames:

Ontology intends to address a lack of shared understanding and the subsequent poor communication among participants, a known problem in IT services. As enterprise IT services originate from the enterprise services that are normally embedded in service supply chains supporting the value co-creation, the shared understanding has to be built based on the service supply chain. From the ontological perspective, the supply chains include the enterprises that supply services, their partners and the service consumers. The enterprises, the partners and the consumers have their own intentions and their own strategies, apart from the common intention of creating values. They depend on each other in supplying the services.

\section{Task I-B: Eliciting IT Service Requirements}

The ontology serves as a meta-model of the role each enterprise in the service supply chains plays. The current Ontology driven elicitation techniques from RE discipline will be explored and possibly extended for IT services. This technique will assist the elicited requirements become sharable, complete and understandable. By conducting a comprehensive review of extant research literature in RE and SOC, we will initially develop a set of service centric evaluation criteria that can be used to assess the suitability of the techniques and methods from RE and SOC research and practice. In our earlier work we have developed a collaborative and situational method and its supporting tool specifically for requirements elicitation workshops. We will examine their utility for eliciting IT services requirements.

\section{Task II: Apply and Extend RE Methods}

We propose to combine $i^{*}$ and the PF approaches to address the challenges in IT services design stated above. Our main objective is to synergize these two analysis approaches and form an integrated treatment for IT services analysis. We would be able to systematically model and analyze both high-level business strategies and values and more concrete level IT service requirements as well as the collaborative work among providers and consumers. We plan to conduct this integration by the following process:

\section{Step 1.Capturing the Business Value Creation Setting}

The integrated analysis approach will first unify the concept of biddable domain [2] in PF with actors in $\mathrm{i}^{*}$ [14] to represent the business value co-creation process. It will answer questions such as: Who will be involved in the service? How will they interact? What will they do? Domain actors, dependencies between them and constraints referring to domains and their dependencies will be identified. The dependencies show interactions between domain actors observable to environment.

\section{Step 2. Understanding Domain Actor Intentions}

This step will attempt to identify why and how the domain actors currently interact by identifying the high level goals of the domain actors, and refining these goals into different interaction relationships. The interactions are shared phenomena in PF and dependencies in $i^{*}$, while the goal concept is inherited from $\mathrm{i}^{*}$.

Step 3. Analyzing Requirements against the Value Creation Setting and Actor Intentions

This step will bring together the information obtained in Task I as well as in the above two steps to form the business requirements. It will utilize various requirements elicitation and analysis techniques to gain a better understanding of real-world problems and consumers' needs. Another important aim of this analysis is to guarantee that the enterprise requirements are consistent, complete and correct. Step 4. Shifting of System Boundary

This step will investigate what role the IT services will play in the value co-creation setting. We will split different roles a domain actor is playing, so that there can be a subset of tasks that the machine will perform. Thus, the decomposition links connecting the nodes are substituted with dependencies between domain actor and IT services. We may then repeat this step, to distribute the tasks taken by the IT services into several subsystems, each of which could be a new individual IT service.

Step 5. Design IT services based on Concrete Interaction

This step will continue the line of analysis to see if the sets of interactions we identified above can be realized by IT services, which suggests the capabilities of IT services. Once the capability model is found for a candidate IT service, we will incorporate generic requirements specification models suggested by the PF to produce the requirement model and specification of the IT service.

\section{Task III Aligning Business Strategies and IT Services}

Our approach for IT service design supports the business strategy/service alignment as these IT services can only be derived from the consumers' service demands, business value creation setting and domain actor intentions. Once these IT services are deployed into practice they may change external/internal environment of the enterprise. That may lead to the changes to the business strategies. This task aims to study the IT service changes in accordance with the changing business setting. It consists of the following two basic elements:

\section{A. Change Management}

First, we will explore and utilize RE change management processes that we have previously developed ([15][16]) to effectively manage the changes in IT services. We will use a change control system to capture and manage change [19].

\section{B. Coordination among Changing Sources}

Change may come from different sources, e.g. the service consumers, the business strategists and the business rules. These sources need to be coordinated to construct a situation in which all the stakeholders can realize mutual gains that is crucial to co-value creation. For supporting the coordination among multiple actors, we will use the negotiation-based approaches to collaborative co-value creation.

\section{Task IV Prototype Toolset}

This task is about designing and implementing a prototype toolset for supporting the whole process of the IT services discovery and design. This toolset will contains tools for 
supporting the alignment analysis between business strategy and IT services. We will also study some real-world cases in particular business domains, e.g. online banking, for evaluating the approach and demonstrating the performance of the toolset.

\section{CONCLUSION}

We believe that the impact of this work will change the way in which IT services are discovered, analyzed and modeled. In particular, methods will be developed for use by IT service providers to ensure that consumer' needs are adequately addressed. There will be two key results that benefit both the IT service providers and consumers. First, the relationship between clients and developers will be clarified and made explicit, allowing for better communication between these two parties. Communication failures are a key reason IT services projects fail. The second result will be clarification of the IT service ProviderConsumer relationship, with the consequence that the outcomes will be much more aligned with true business strategy and goals. This research will provide significant benefit to the growing IT service industries. It will result in improved services (and hence improved competitiveness of this industry in a global market) that better satisfy specific business objectives. Development cycles will shorten, as the likelihood of redevelopment being required will be less. Finally, the models, methods and tools that will be developed will facilitate the transition of traditional "bricks and mortar" organizations into the new economy, as the outcomes of this research will provide a clear approach for identifying and clarifying the evolving business domain and the requirements of companies developing Services within this new domain. This project will also develop and deliver innovative methods and workable tools. The project will highlight a spectrum of new problems and their solutions, which will significantly extend and broaden the RE methods and technologies into a new field of IT service design.

\section{REFERENCES}

[1] IBM, Service Science Management and Engineering (SSME), http://www.ibm.com/developerworks/spaces/ssme.

[2] M. Jackson, Problem Frames: Analysing and Structuring Software Development Problems. Addison-Wesley, 2001.

[3] Office of Government Commerce, ITIL Service Design, TSO, London, 2007.

[4] A. Wegmann, G. Regev, G-A. Garret, and F. Marechal, Specifying Services for ITIL Service Management, in Proc. Int Workshop on
Service-Oriented Computing Consequences for Engineering Requirements (SOCCER'08), Barcelona, Spain, September 08, 2008.

[5] ISO/IEC, 20000-1 IT Service Management - Part 1, ISO/IEC, Geneva, Switzerland, 2005.

[6] ISACA, www.isaca.org/cobit/, accessed February 2009.

[7] E. Gottesdiener, Requirements by Collaboration: Workshops for Defining Needs. Addison-Wesley. 2002.

[8] N. Maiden N, S. Manning, S. Robertson, J. Greenwood, Integrating creativity workshops into structured requirements processes. In Designing Interactive Systems, Cambridge, MA, August 1-4, 2004.

[9] C. Coulin, D. Zowghi, AEK Sahraoui, AEK, A Situational Method Engineering Approach to Requirements Elicitation Workshops in the Software Development Process, Journal of Software Process: Improvement and Practice, vol. 11, pp. 451-64, 2006.

[10] C. Coulin, D. Zowghi, AEK Sahraoui, MUSTER: A Situational Tool for Requirements Elicitation, Book Chapter in "Artificial Intelligence Applications for Improved Software Engineering Development: New Prospects", edited by Farid Meziane and Sunil Vadera, published by IGI Global, 2009.

[11] P. Wang, Z. Jin, L. Liu, and G. Cai, Building Towards Capability Specifications of Web Services Based on an Environment Ontology, IEEE Transactions on Knowledge and Data Engineering, Vol.20, No.4, 547-561, 2008

[12] P. Wang, Z. Jin, L. Liu, Context Ontology-based Capability Specification for Web Service Discovery, Proceedings of $8^{\text {th }}$ International Conference on Formal Engineering Methods (ICFEM 2006), LNCS 4260:185-205, 2006

[13] B. Wu, Z. Jin and B. Zhao, A Modeling Approach for ServiceOriented Application Based on Extensive Reuse, Proceedings of International Conference on Web Services (ICWS2008): 754-757, 2008

[14] E. Yu, Towards Modeling and Reasoning Support for Early-Phase Requirements Engineering. Proceedings of the 3rd IEEE International Symposium on Requirements Engineering (RE'97): 226-235, 1997.

[15] Gervasi V., Zowghi D., Reasoning about Inconsistencies in Natural Language Requirements, the ACM Transactions on Software Engineering and Methodology (TOSEM), 14(3): 277-330, July 2005.

[16] D. Zowghi, V. Gervasi, On the Interplay Between Consistency, Completeness, and Correctness in Requirements Evolution, Journal of Information and Software Technology, Vol 46(11), 763-779, 2004.

[17] K. Mu, Z. Jin, and D. Zowghi, A Priority-based Negotiations Approach for Handling Inconsistencies in Multi-perspective Software Requirements, Journal of Systems Science and Complexity, 21(4): 566-588, 2008.

[18] B. Wang, W. Zhang, H. Zhao, Z. Jin and H. Mei, A Use Case Based Approach to Feature Models' Construction, Proceedings of the $17^{\text {th }}$ International Conference on Requirements Engineering (RE'09): 121130,2009

[19] Nurmuliani, D. Zowghi, Williams S., "Characterising Requirements Volatility: An empirical Analysis", Proceedings of the $4^{\text {th }}$ International Symposium on Empirical Software Engineering (ISESE), Noosa, Australia, November 2005

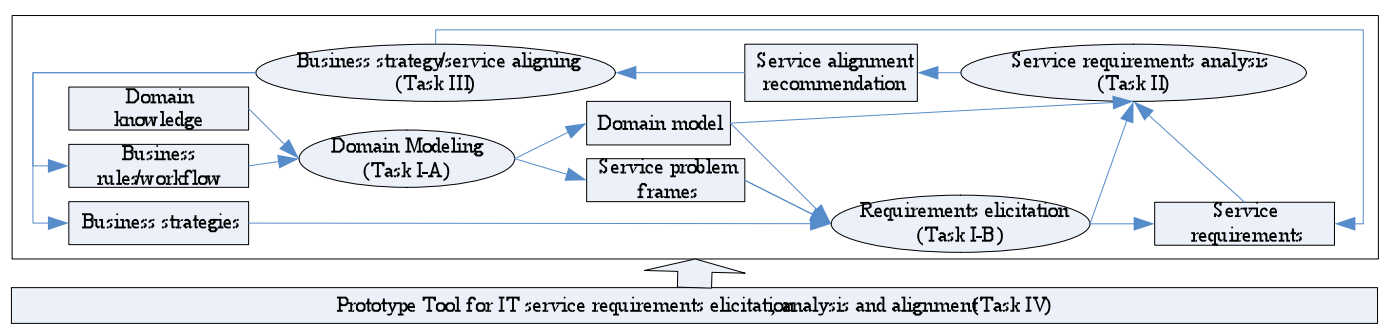

Figure 1. Overview of the research tasks 